\title{
Alineamiento Estratégico Sectorial: Caso de Estudio Aplicado a una Universidad Chilena
}

\author{
Ana M. Barra \\ Universidad del Bío-Bío, Facultad de Ciencias Empresariales, Avda. Collao 1202, CP 4051381 \\ Concepción-Chile (e-mail: abarra@ubiobio.cl).
}

Recibido Jul. 29, 2014; Aceptado Oct. 10, 2014; Versión final recibida Dic. 1, 2014

\begin{abstract}
Resumen
En el presente artículo se analiza la estructura del plan estratégico institucional y los planes sectoriales o de las unidades que conforman una universidad. La situación de la Universidad del Bio Bio, ubicada en Concepción (Chile) es tomada cono caso de estudio. A través de una metodología cualitativa y estructurada, tanto de aplicación del conocimiento de las temáticas, como participativa en la confección y retroalimentación de dichos planes, se evidencia que existen distintos estados de avances de estos procesos de planificación estratégica. Este desnivel de avance afecta la rapidez del logro del alineamiento sectorial al institucional. Para lograr el alineamiento de los planes de las distintas unidades (Facultades, Departamentos, Escuelas) al Plan de Desarrollo Corporativo se adoptó en forma exitosa un modelo propuesto por el estamento directivo de la universidad.
\end{abstract}

\section{Sectorial Strategic Alignment: Case applied to a Chilean University}

\begin{abstract}
In this article, the structure of the corporate strategic plan and the sectorial plans of the units that compose a university are analyzed. The situation of the University of Bio-Bio in Concepción (Chile) is considered as a study case. Using a structured qualitative methodology, both applying knowledge of the subjects and also participating in the preparation and feedback of these plans, it was found that there are degrees of advance of these processes of strategic planning. This different progress affects the efficiency in achieving sectorial alignment to the corporate plans. To achieve the alignment of plans from the (Faculties, Departments, Schools) to the Corporate Development Plan a model proposed by the university administration was successfully implemented
\end{abstract}

Keywords: strategic alignment, strategic planning, development plans, university administration 


\section{INTRODUCCIÓN}

El continuo cambio de escenarios a los cuales se ve enfrentada la organización y la constante preocupación de mantener o aumentar su posicionamiento competitivo, crear más valor, destacar su sello diferenciador frente a la competencia entre otros, son razones importantes a la hora de cuestionarse la coherencia que existe entre la visión, misión, objetivos y estrategias de las distintas unidades de una universidad como son las facultades, los departamentos y carreras, con el alineamiento institucional de ella. Los cambios que ocurren en el entorno como los políticos, económicos, tecnológicos pueden llevar a la organización a enfrentar desafíos importantes en el funcionamiento de ellas, de modo que es de suma relevancia abordar de manera constante este tema si se quiere lograr mejores rendimientos en los resultados de ella.

La investigación en la educación superior ha proporcionado nuevas perspectivas y recomendaciones para la gestión de las instituciones de educación superior (Brown II, 2010), es así como hoy en día la revisión de los instrumentos de gestión de las organizaciones, como son los planes generales y sectoriales de las universidades son de suma importancia para ver los impactos en los resultados de ella y ajustar y validar nuevas propuestas metodológicas para la institución. Por otro lado, dado el centralismo de los servicios públicos a través del mundo y las continuas reformas experimentadas, tales como la modernidad del gobierno, a partir de los años 90 en Chile, es de suma importancia visualizar cómo se traduce esta idea en el tema del alineamiento estratégico de una institución de educación superior, pública (Ferlie, 2001). Sumado a lo anterior, algunos autores, como Deem y Brehony (2007), hablan de "New Managerialism", para referirse a su rol en la reforma de la educación superior.

\section{Alineamiento estratégico}

Senge (1999), plantea que la relación entre las partes es más importante que las partes individuales por sí mismas. Esto quiere decir, que el desempeño de un sistema integrado, es más relevante que el desempeño de las partes independientes, o dicho en otras palabras, que para lograr el desempeño del sistema, es necesario alinear o sincronizar el desempeño de cada uno de los componentes individuales de dicho sistema, como son, departamentos y áreas funcionales de la empresa. Este proceso de sincronización debe ser continuo a fin de garantizar los objetivos y la optimización de los recursos de la organización, entre otros.

Según la Real Academia Española, alineación es "acción y efecto de alinear y vincular a otros a una tendencia ideológica, política, etc". Sumado a lo anterior se puede decir que la construcción del cuadro de mando permite alinear las directrices estratégicas, como visión, misión con los objetivos y estrategias a fin de establecer las brechas y ajustes necesarios una vez transcurrido un tiempo determinado de modo que los cambios que se deben realizar lleven a la organización a la optimización de recursos y al aumento de valor para clientes y actores involucrados. En términos simples se dice que una organización está alineada cuando los objetivos de sus departamentos son coherentes con los objetivos estratégicos de la organización.

En el Informe de la Dirección Estratégica de las Universidades Españolas (2009), se plantea que una vez terminada la etapa de la Formulación de la Estrategia, se debe llevar a cabo el Despliegue, en la que los distintos ámbitos de la universidad trabajen en desarrollar su propia Estrategia, que sea totalmente coherente y alineada con la institucional. El reto de desplegar la estrategia, es decir, lograr que llegue a ser realidad, es una fase compleja en la que se unen la necesidad de comunicar de una manera efectiva la estrategia, con la de implicar a los siguientes niveles en el desarrollo del despliegue y la de llegar hasta las acciones mediante proyectos concretos con el uso de indicadores medibles. Para profundizar la forma en que se despliega la estrategia a las unidades internas, es necesario reflexionar sobre cómo se hace operativa la estrategia institucional.

En cuanto a los elementos alineados con la estrategia institucional, el Informe plantea que el alineamiento de los factores y elementos que integran los sistemas que permiten la gestión de una universidad juega un papel importante en el logro de la implantación de la estrategia definida. Se plantean algunos elementos para analizar el grado de alineación de la estrategia de las unidades con la estrategia Institucional como el presupuesto anual, gestión de la calidad, política de personal, sistemas de seguimiento, sistemas de información, gestión por procesos, responsabilidad social corporativa, entre otros.

Los autores Kaplan y Norton (2008), plantean la importancia del diseño de un Sistema de Gestión para alinear la estrategia de las unidades de negocios con las operaciones y, dado que la mayoría de las organizaciones constan de múltiples unidades de negocios y de soporte, el sistema de gestión también debe abordar cómo se integra la estrategia en las diversas unidades organizacionales. Además de alinear 
las unidades organizacionales con la estrategia, el sistema debe alinear con ella también a los trabajadores. Dichos autores plantean un sistema de alineación que consta de tres etapas: (i) alinear las unidades de negocios; (ii) alinear las unidades de soporte; y (iii) alinear a los trabajadores.

1. Alinear las unidades de negocios, cuyo objetivo es desdoblar e incorporar la estrategia corporativa a las estrategias de las unidades de negocios. También en este aspecto hay que considerar que las barreras más típicas de encontrar son situaciones donde las estrategias de las unidades de negocios se desarrollan y aprueban de manera independiente, sin la guía de una perspectiva, observándose de esta manera, una falta de integración entre las unidades de negocios.

2. Alinear las unidades de soporte, cuyo objetivo es garantizar que cada unidad de soporte tenga una estrategia que mejora el desempeño de las estrategias de la organización y las unidades de negocios. Las barreras que se pueden encontrar son que a las unidades de soporte se les trata como "centros de gastos discrecionales" con objetivos para minimizar los costos en vez de soportar las estrategias de las unidades de negocios y la compañía.

3. Alinear a los trabajadores, cuyo objetivo es que todos comprendan la estrategia y estén motivados para ejecutarla de manera exitosa. En este caso la barrera apunta a que la mayoría de los empleados no conocen o no entienden la estrategia. Sus objetivos e incentivos se focalizan en el desempeño táctico y local y no en los objetivos estratégicos.

Pettit (2003), se refiere a diseño institucional como la creación de acuerdos institucionales totalmente nuevos como también, y primordialmente, al examen y reelaboración cotidianos de los ya existentes. $Y$ agrega diciendo que una institución es buena en la medida en que fomente el cumplimiento, en la medida en que asegure que los agentes se ajustan (o se abstienen de hacerlo) a las pautas de conducta prescritas (o proscritas) por la misma.

\section{Planificación Estratégica}

El autor Fernández (2004), se refiere a la planificación estratégica como el proceso que nos indica las acciones a emprender para conseguir los fines, teniendo en cuenta la posición competitiva relativa, y las previsiones e hipótesis sobre el futuro. Por otro lado Larrosa (1995), afirma que la planificación empresarial "es un proceso formalizado de toma de decisión que elabora una representación deseada del estado futuro de la empresa y especifica las modalidades de puesta en práctica de esta voluntad".

Para los autores Anthony y Govindarajan (2003), la planificación estratégica puede ser definida como el proceso de decidir sobre los programas que la organización va a acometer y sobre la cantidad aproximada de recursos que se asignará a cada programa para los siguientes años. Además los autores hacen una distinción entre formulación de estrategias y planificación estratégica. La primera se refiere al proceso de decisión sobre las nuevas estrategias, la segunda se ocupa de las decisiones necesarias para implantar las estrategias elegidas y hacer que funcionen. En cambio Aceves (2004), la define como la "identificación sistemática y sistémica de las oportunidades y peligros futuros que, combinados con las fortalezas y debilidades, proporcionan una base para la toma de decisiones ventajosa en el presente para aprovechar 0 crear las oportunidades, y evadir, evitar o transformar los peligros en oportunidades". Taylor (1979), la conceptúa como "el proceso de elegir el mejor camino entre dos puntos: los negocios de hoy son el punto A; los negocios del mañana, el B. La planeación estratégica es el examen de las rutas más prácticas entre estos dos puntos, a fin de que se elija el curso óptimo. La verdad es que la buena planeación va aún más allá, porque también ayuda a establecer la ubicación del punto B".

Sin lugar a dudas el autor Fernández (2004) deja claro que en el proceso de planificación estratégica son importantes las formas en que se consiguen los fines, en este trabajo, para lograr el alineamiento son importantes las políticas establecidas por la universidad, ya que ellas contribuyen a lograr mejores resultados en la organización, al tener claridad de las acciones que desarrolla cada unidad, como también de las directrices, objetivos, estrategias y formas de control de la institución y a la cual las unidades, como departamentos y carreras deben mirar y alinearse. De este modo es factible que este alineamiento resulte ser un factor diferenciador en relación a otras instituciones.

\section{Ventaja Competitiva}

Según Grant (1996), el crear ventaja competitiva implica gestionar recursos internos y capacidades de manera que hagan frente a oportunidades externas y el mantenimiento de la ventaja competitiva depende de las características y disponibilidades de los recursos y capacidades. 
Ahora los autores Teece et al (1997), afirman que una ventaja competitiva constituye una destreza o habilidad especial que logra desarrollar una empresa, que permite desarrollar uno o más factores diferenciadores en sus operaciones, sus productos y/o sus servicios, que la coloca en una posición de preferencia a los ojos del mercado, ya que sus integrantes perciben la propia organización y/o sus productos o servicios como algo único y determinante en el proceso de decisión de compra. Una última aproximación que permite visualizar fuentes de Ventajas competitivas corresponde a la Perspectiva de los Recursos (Teece et al., 1997), ellos argumentan que la aproximación de los recursos enfatiza la construcción de la ventaja competitiva a través de capturar rentabilidad intraempresarial provenientes de ventajas eficientes en los niveles de la firma.

\section{Estrategia}

Para Ohmae (2004), la estrategia empresarial, en una palabra, es ventaja competitiva. El único propósito de la planificación es permitir que la empresa obtenga, tan eficientemente como sea posible, una ventaja sostenible sobre sus competidores. La estrategia corporativa supone, así, un intento de alterar las fortalezas relativas de la compañía para distanciarse de sus competidores de la manera más eficiente. Thompson y Strickland (1999) "La estrategia de una organización consiste en las acciones combinadas que ha emprendido la dirección y qué pretende para lograr los objetivos financieros y estratégicos y luchar por la misión de la organización. Esto a la larga nos va a ayudar a cómo lograr nuestros objetivos y cómo luchar por la misión de la organización".

Johnson y Scholes (2001) "Estrategia es la dirección y el alcance de una organización a largo plazo, y permite conseguir ventajas para la organización a través de su configuración de recursos en un entorno cambiante, para hacer frente a las necesidades de los mercados y satisfacer las expectativas de los stakeholders. En opinión de Rodríguez (1998), la estrategia "define el modo o plan de acción para asignar recursos escasos con el fin de ganar una ventaja competitiva y lograr un(os) objetivo(s) con un nivel de riesgo aceptable". Según el autor Grant (1996), la estrategia es la materia unificadora que da coherencia y sentido a las decisiones individuales de una organización o una persona. Su principal objetivo es facilitar el éxito de la empresa, guiando las decisiones directivas hacia la creación y el mantenimiento de una ventaja competitiva. Richardson, y Richardson, (1996), la definen de manera más simple, señalando que la estrategia "es un medio para alcanzar un fin". Para Chandler (1966), la definición de las metas y objetivos a largo plazo de una empresa, y la adopción de acciones y la asignación de los recursos necesarios para la consecución de estos objetivos.

Sin embargo para Quinn (1980), la estrategia es "el modelo o plan que integra los principales objetivos, políticas y sucesión de acciones de una organización en un todo coherente. Una estrategia bien formulada ayuda a ordenar y asignar los recursos de una organización de una forma singular y viable basada en sus capacidades y carencias internas relativas, en la anticipación a los cambios en el entorno y en las eventuales maniobras de los adversarios inteligentes. Hermida (1992), concibe la estrategia como "la adaptación de los recursos y habilidades de la organización al entorno cambiante, aprovechando sus oportunidades y evaluando los riesgos en función de los objetivos y metas". En resumen la estrategia es esencialmente la forma o el camino que la empresa sigue para adaptarse al contexto y lograr sus objetivos.

De las definiciones descritas anteriormente se destacan en particular los objetivos y metas que toda compañía persigue e intrínsecamente la importancia que las estrategias representan para su consecución. Las estrategias suelen ser diseñadas y a la vez establecidas por los distintos niveles dentro de la organización, en este caso el institucional, que sirve de base para la formulación de las estrategias de los departamentos y carreras. Importante será observar si éstas se manifiestan en forma explícita o implícita dentro del proceso de planificación estratégica.

\section{METODOLOGÍA}

En relación a lo estudiado y considerando los objetivos planteados, además de la revisión bibliográfica, se puede determinar que nuestro estudio tiene carácter exploratorio, debido a que se trata de un estudio que no se ha realizado antes. Si bien es cierto se han seguido algunos pasos metodológicos del estudio realizado por Anderson et al (1999) a las universidades australianas, este estudio tiene un enfoque distinto, logrando establecer un análisis distinto de la planificación estratégica de una institución de educación superior. Además de tener un carácter exploratorio, este estudio tiene la particularidad de ser de tipo cualitativo, lo que permite adoptar criterios e impresiones en relación al tema tratado. Junto a lo anterior, este estudio también posee carácter de descriptivo ya que se detallan todos sus componentes principales, como son alineamiento estratégico, planificación estratégica y estrategia. (Caiceo y Mardones, 1998) 
Ahora bien, desde el punto de vista científico, describir es medir, por ello en los estudios de tipo descriptivo se selecciona una serie de variables y se mide cada una de ellas independientemente, para así describir lo que se investiga En esta clase de estudio se debe ser capaz de definir qué se va a medir y cómo lograr precisión, asimismo es necesario identificar quiénes estarán incluidos en la medición. La descripción puede ser más o menos profunda, pero en cualquier caso se basa en la evaluación de uno o más atributos del fenómeno descrito. (Hernández, Fernández-Collado y Baptista, 2006). Para explicar la metodología utilizada para la confección del Plan de Desarrollo de la Universidad y los planes sectoriales, se analizarán los elementos que intervienen en la confección de los planes estratégicos, y son los insumos, los actores involucrados y el proceso aplicado.

\section{Insumos requeridos}

Para la formulación del Plan General de la Universidad se requieren los siguientes insumos: Un Comité de Planificación, que se constituye al interior de cada Facultad, con la participación de ciertos actores relevantes (académicos) en el proceso de definición de algunos aspectos del proceso de planificación, más los profesionales que pertenecen a la Dirección General de la Universidad, que son los encargados de elaborar el Plan estratégico a nivel Corporativo, de comunicar y dar a conocer a las unidades dicho Plan, de entregar los lineamientos o el formato para la construcción de los Planes estratégicos de las Unidades y por último, revisar y retroalimentar los planes de las Unidades una vez construidos.

En cuanto a los insumos del Plan Estratégico Corporativo o Institucional, se detallan los siguientes elementos: directrices estratégicas (Visión, Misión), análisis del entorno, análisis interno, diagnóstico estratégico (FODA), objetivos generales, objetivos específicos, resultados esperados, verificadores, metas y presupuesto. En lo referente al análisis del entorno de la universidad hay que considerar los aspectos de aquellas funciones esenciales de una universidad como son, la Docencia, la Investigación, el Posgrado, la Vinculación con el Medio y la Gestión Institucional, las cuales ya se encuentran incluidas, como líneas estratégicas u objetivos estratégicos en el Plan Estratégico Institucional. En cuanto a las variables del análisis interno hay que considerar las Académicas y las Organizacionales. En lo referente a las Académicas, se analizan aspectos referidos a: Personal académico; Alumnos, Planes: Estudio e Investigación; Proceso de Enseñanza-Aprendizaje, Investigación; Infraestructura: aulas, equipo; Impacto y Vinculación.

En relación a las variables Organizacionales, hay que analizar aspectos referidos a la Normatividad, Gobierno, Planeación y Coordinación, Apoyo Académico, Apoyo Administrativo, Recursos Financieros, Recursos Humanos, entre otros. Hay que destacar que los insumos usados en la construcción de los planes estratégicos de las universidades varían de una a otra y no existe uniformidad de ellos.

\section{Actores involucrados}

Para la formulación del Plan General de la Universidad, se recomienda una participación triestamental en el análisis interno que incluye a los Académicos, los Administrativos y los Estudiantes. De igual modo se sugiere una participación de la sociedad en el análisis externo, que incluye a ex alumnos, empleadores, líderes de opinión y políticos, entre otros. Los académicos como artífices, junto con los directivos del cumplimiento de los planes establecidos; los administrativos que deberán cumplir con las políticas y procedimientos de la universidad y los estudiantes que estarán inmersos en una carrera que les ofrece claridad de sus directrices estratégicas, entre otros elementos importantes, que les servirá para la elección y permanencia en dicha carrera y universidad.

\section{Proceso aplicado}

El proceso de formulación del Plan General de Desarrollo Universitario (2011) se realiza en seis etapas, según se muestra en la figura 1.

La etapa 1 comprende la evaluación del PGDU anterior, la propuesta de la metodología para el Plan del próximo período y la presentación de ésta al Comité de Planificación de la universidad para revisión y validación.

La etapa 2 comprende la comunicación a la comunidad universitaria sobre el nuevo proceso de formulación del PGDU; la preparación y desarrollo de talleres ampliados para evaluar y definir misión y visión; la redacción definitiva de misión y visión; la preparación de encuesta de valores; la aplicación de encuesta vía Web y la definición de valores compartidos. 


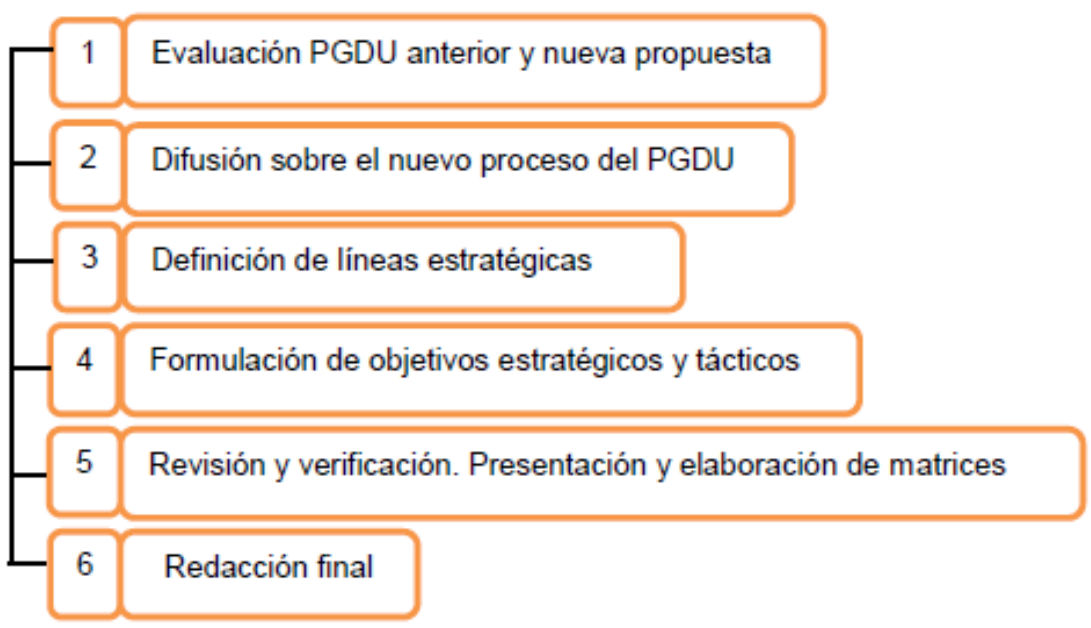

Fig. 1: Esquema Proceso de formulación del Plan General de Desarrollo Universitario (PGDU).

La etapa 3 comprende la definición de líneas estratégicas; la convocatoria para la formación de comisiones de trabajo por línea estratégica; la preparación de talleres FODA por parte de las comisiones de trabajo; el desarrollo de talleres y la consolidación de los FODAS con el institucional.

La etapa 4 comprende la formulación de los Objetivos Estratégicos a partir del FODA y la formulación de los Objetivos Tácticos para cada uno de los objetivos estratégicos.

La etapa 5 comprende la revisión de los objetivos tácticos para verificar consistencia entre éstos, los objetivos estratégicos, la misión y la visión institucional; la elaboración de las matrices de planificación para cada objetivo, definiendo metas, indicadores y responsables; la definición del modelo de seguimiento del Plan General y la presentación de las matrices de planificación al Comité de Planificación para revisión y validación.

La etapa 6 comprende la redacción del documento final, la presentación a la Junta Directiva, instancia máxima de la universidad, para su aprobación y la presentación y difusión del nuevo PGDU a la comunidad universitaria

En cuanto a los niveles donde se realiza la Planificación Institucional, se formula en tres niveles que son: el nivel estratégico, el nivel programático y el nivel operativo. En el nivel estratégico se establecen la visión, misión, líneas estratégicas, objetivos estratégicos, objetivos tácticos y metas, del Plan General de Desarrollo, que se formula en la Dirección General de Planificación de la Universidad. En el nivel programático se establecen las líneas estratégicas o programáticas, los Objetivos Generales, los Objetivos Específicos y las metas, dando como resultado la obtención de los Planes de Desarrollo Programáticos que se formulan a nivel de Vicerrectorías, Direcciones Generales y Facultades. En el nivel operativo se formulan Objetivos Generales, Objetivos Específicos y metas, lo que da como resultado los Planes de Desarrollo Operativos, que se formulan en los Departamentos y Escuelas.

En cuanto al período en que se debe formular el Plan, corresponde a cuatro años de vigencia en conjunto con los planes de Facultades, Departamentos y Escuelas. Específicamente para la construcción de un plan estratégico departamental se deben considerar los siguientes insumos: En primer lugar contar con la Asesoría técnica del Comité de Planificación constituido al interior de la facultad, quien dará los lineamientos que deberá contener el plan; en segundo lugar se debe contar con el Plan Estratégico de la Facultad y en tercer lugar contar con la matriz para elaborar este plan, que es entregada por la Dirección de Planificación institucional y que contiene elementos similares a los de facultad tales como visión, misión, objetivos generales, resultados esperados, verificadores, objetivos específicos, metas, indicadores, responsables, costos y fuentes de financiamiento. El Plan departamental, además de estar alineado a las directrices de la facultad debe ser consecuente con algunos de los objetivos institucionales tales como de Docencia, Investigación y Vinculación con el Medio, entre otros.

En cuanto a los actores involucrados en este proceso, se sugiere la participación de todos los docentes que pertenecen al departamento como también de aquellos que prestan servicios a éste en conjunto con el Director de Departamento. En la realidad, y referido al proceso de los planes departamentales, se encontró que diferían del institucional, dado que se habían formulado para tres años y debían ser para cuatro, es decir para igual período que el formulado para la universidad, por tanto un primer criterio fue partir con la 
reformulación de los planes estratégicos a cuatro años. Posteriormente la Facultad inicia su proceso de revisión del plan ya que éste sería la base de los planes estratégicos de los departamentos y escuelas.

El trabajo de la facultad se inició considerando como insumos los explicados anteriormente y que corresponden al Plan Estratégico General Universitario, 2011-2014 (PGDU), además se consideraron el Modelo Educativo de la Universidad, el Informe de Autoevaluación Institucional, el Plan de Mejoramiento, los Instructivos Presupuestarios de la Vicerrectoría de Asuntos Económicos y los Planes de Mejoramiento de las carreras. Este trabajo se inicia con el asesoramiento técnico y especialista de un comité de Planificación constituido al interior de la facultad, compuesto por tres docentes y cuyas funciones se detallan a continuación: a) Asesora a la Facultad proporcionando apoyo técnico a los procesos de planificación de los niveles operativos; b) Colabora con el Decano en la construcción del Plan Programático de la Facultad; c) Actúa como nexo entre la Facultad y la Dirección General de Planificación y Estudios; y d) Adicionalmente, el comité participa de tres talleres de capacitación técnica que le permitirán asesorar a las unidades.

Una vez realizado los cambios al plan de la facultad, este es consensuado al interior de ella con la participación de todos los docentes y directores de departamento y escuela a objeto de recibir observaciones e ideas que lo complementen y posteriormente entregarlo como documento base, para que los departamentos reformulen y revisen su plan según las directrices y objetivos contemplados en él.

A lo anterior hay que agregar que el plan de la facultad debe estar alineado a las cinco líneas estratégicas de la universidad, no así a todos sus objetivos, solamente a los atingentes a su desarrollo. Una vez terminado y alineado el plan estratégico de la facultad al plan de la universidad, son los departamentos los que inician el trabajo de alineamiento. En este caso el alineamiento debe ser correspondiente con la misión y visión de la facultad y con los objetivos de ella que son a su vez correspondiente con algunos objetivos estratégicos de la universidad. En el caso de las carreras, deben formular su plan estratégico tomando como base el plan departamental principalmente, aunque siempre considerando el plan de la facultad. En la Figura 2, se presentan los conceptos y procesos llevados a cabo para la confección de los Planes Estratégicos.

\section{RESULTADOS}

Para iniciar el análisis del grado de alineamiento existente de los planes estratégicos, tanto de los departamentos como de las carreras con el plan estratégico institucional, se detectó que las unidades tenían formulados visión, misión, objetivos, acciones para lograr esos objetivos, pero que muchas veces no correspondían con los institucionales. En cuanto a mecanismos de seguimiento y control no existía en ninguna de ellas. En otras palabras, los planes estratégicos estaban formulados principalmente por directrices estratégicas y algunas acciones para lograr esos planes. El período de tiempo también era desigual, algunos correspondían a tres años y otros cuatro. Por tanto se hacía evidente y altamente necesario implantar alguna metodología para la elaboración de los planes de departamentos y carreras y hacerlos corresponder, de esta manera con el Plan institucional.

El modelo que se aplicó para desarrollar los planes de facultad, departamentales y de carreras contenía los siguientes elementos: Visión-Misión-Objetivo general-Objetivo específico-Indicador-Meta-ResponsableCosto total-Fuente de Financiamiento. Y estos debían estar alineados principalmente hacia la correspondencia del objetivo general y específicos con el objetivo estratégico y línea estratégica de la universidad. En la Figura 3 se puede visualizar esta relación. Una vez formulados los planes de desarrollo de la facultad, del departamento y de las carreras, se entregan a la Dirección General de Planificación de la Universidad, quién aplica un formulario de cotejo o de revisión de los planes estratégicos sectoriales con el institucional, abordando los siguientes puntos: (a) Aspectos generales como nombre de la unidad, responsable de la unidad, nombre del revisor, fecha de entrega, días de revisión; y (b) Aspectos a evaluar como: ficha de presentación, organigrama de la unidad, visión de la unidad, coherencia con la visión institucional, misión de la unidad, coherencia con la misión institucional, identificación de fortalezas, identificación de debilidades, identificación de oportunidades, identificación de amenazas, identificación de problemas o desafíos, identificación de causas, objetivos generales, resultado esperado, verificador, objetivo específico, identificación de meta, coherencia de las metas, verificador, observaciones generales. Una vez revisados los planes, son retroalimentados por la Dirección General de Planificación utilizando el formulario detallado anteriormente, donde se detallan tres alternativas de observaciones: 1) Aceptado sin observaciones; 2) Aceptado con observaciones menores; y 3) Corregir según observaciones realizadas

Además de estos tres escenarios se retroalimenta cada una de los ítems contenidos en los distintos planes de desarrollo explicados en el formulario. Se sugiere realizar esta exhaustiva revisión de los planes de desarrollo de las distintas unidades en forma anual a fin de facilitar el cumplimiento, revisión y ajustes del mismo. 


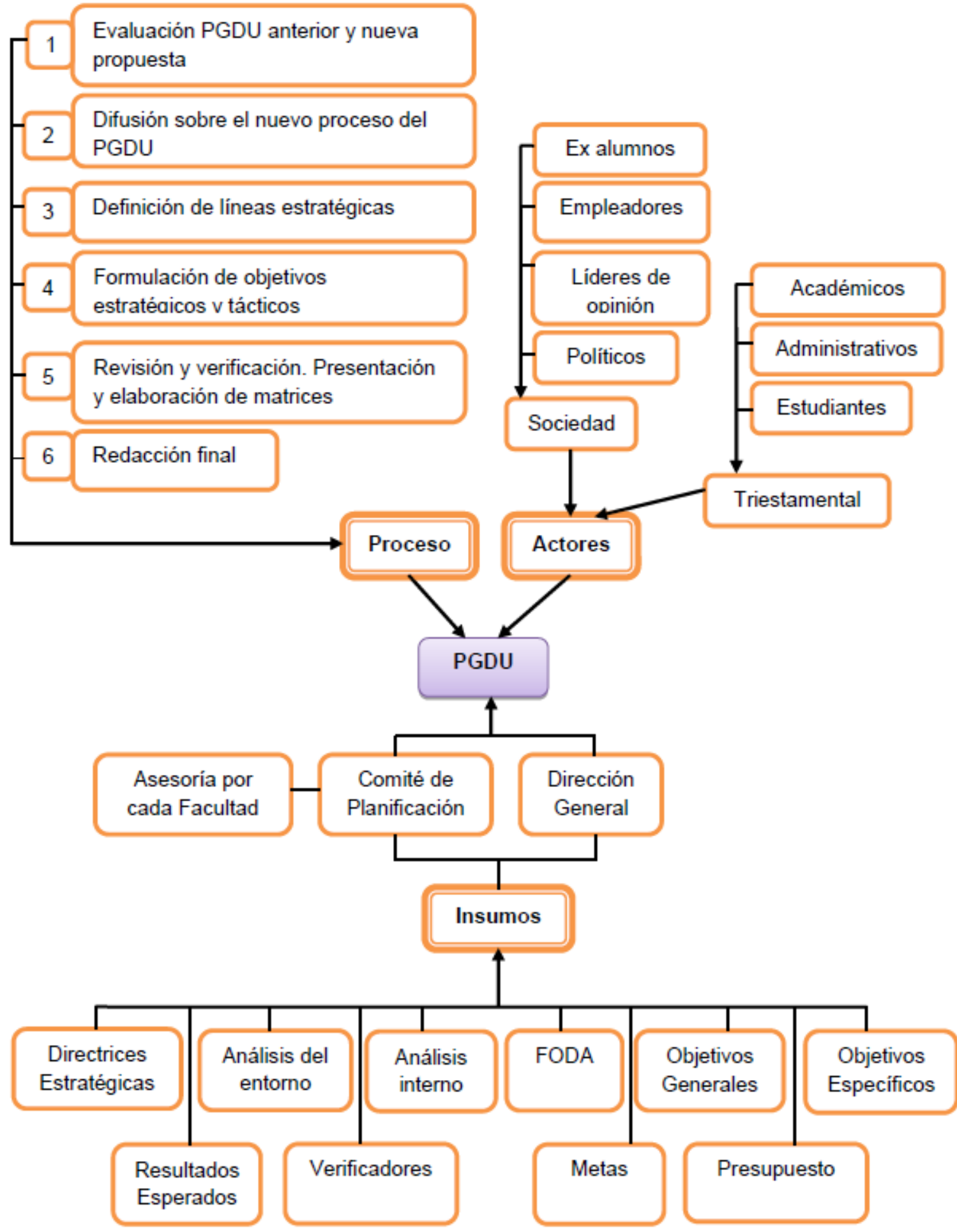

Fig. 2: Esquema de la Metodología.

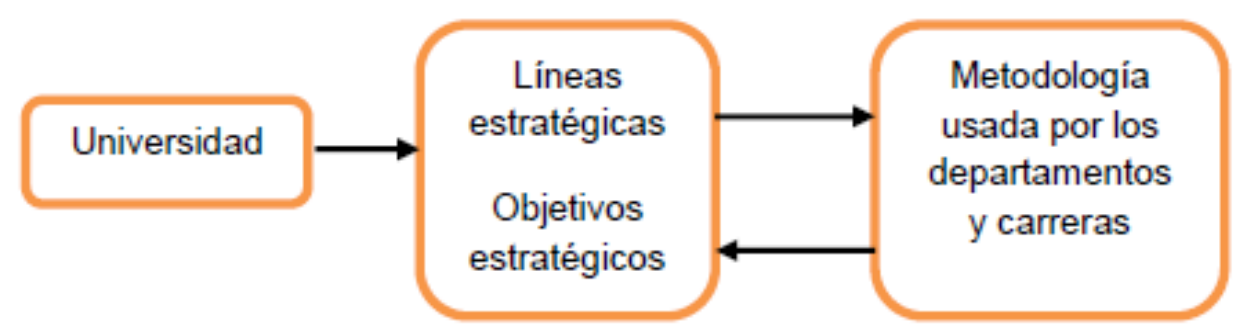

Fig. 3: Esquema de alineamiento. 


\section{CONCLUSIONES}

De los resultados obtenidos y de su análisis, y considerando que el objetivo central de este estudio fue realizar un alineamiento estratégico de los Planes de Desarrollo de las diferentes unidades que la componen, al Plan de Desarrollo Institucional o Corporativo, se puede concluir que:

En relación a los insumos utilizados para la formulación de los planes estratégicos de las unidades, estos debieran ser todos iguales, sin embargo al momento de realizar el estudio se concluye que no lo es, dado que el grado de elaboración de los planes de las unidades obedecían al diagnóstico y a algunas acciones para el logro de los objetivos.

La Planificación estratégica de la Universidad presentaba aspectos que no eran explícitos, como las estrategias, lo que hizo que todos los planes no la manifestaran en sus planes.

Gracias al nuevo modelo de planificación estratégica impuesto por la universidad se logró el alineamiento de los planes estratégicos de las unidades con el plan estratégico de la Universidad.

Y por último, para realizar el seguimiento del alineamiento, se implementó un software para optimizar tiempos y recursos, lo que está en evaluación aún.

\section{REFERENCIAS}

Aceves, V., Dirección Estratégica. Primera Edición, Editorial McGraw-Hill, México DF, México pp. 1-367 (2004)

Anderson, R., Hair, J., Tatham, R., y Black, W., Análisis Multivariante. Quinta Edición, Editorial Prentice Hall, Madrid, España (1999)

Anthony, R. y Govindarajan, V., Sistemas de Control de Gestión. Décima Edición, Editorial McGraw-Hill, Madrid, España (2003)

Barra, A., Influencias del Aprendizaje Organizacional en la obtención de Ventajas Competitivas: aplicación al sector industrial pesquero chileno exportador de salmón a la Unión Europea. Tesis Doctoral, Universidad Politécnica de Cataluña, Dep. de Organización de Empresas, Facultad de Ingeniería Industrial (2007)

Brown II, M.C., Organization y Governance in Higher Education, Quinta Edición, Editorial Pearson Custom Publishing, Boston, Estados Unidos (2010)

Caiceo, J. y Mardones L., Elaboración de tesis e informes técnico-profesionales, pp. 1-258, Editorial Jurídica ConoSur Ltda., Santiago, Chile (1998)

Chandler, A., Strategy and Structure. Anchor Books Edition, Editorial Doubleday, New York, Estados Unidos (1966)

Deem R. y Brehony K., Management as ideology: the case of "new managerialism" in higher education, Oxford Review of Education, Vol. 31, No 2, pp. 217-235, (2007)

Ferlie, E., McLaughlin, K., y Osborne, S., New public management: current trends and future prospects, Primera Edición, Editorial Routledge, Londres, Reino Unido (2002)

Fernández, A., Dirección y Planificación Estratégicas en las empresas y organizaciones, pp. 1- 219, Ediciones Díaz de Santos S.A., Madrid, España, (2004)

Grant, R., Prospering in Dynamically Competitive Environments: Organizational Capability as Knowledge Integration. Organization Science, Vol. 7, No 4, pp. 375-87, (1996)

Hermida K., Administración y Estrategia, Cuarta Edición, Ediciones Macchi, México DF, México (1992)

Hernández, R., Fernández-Collado, C. y Baptista, P. Metodología de la investigación, Cuarta Edicióm, pp.150-155, Editorial McGraw-Hill, México DF, México (2006)

Informe de la Dirección Estratégica en las Universidades Españolas Cátedra Unesco de Dirección Estratégica. Documento Institucional (2009) 
Johnson, G. y Scholes, K. Dirección Estratégica. Quinta Edición, pp. 1- 716, Pearson Education S.A., Madrid, España (2001)

Kaplan, R y Norton, D. The Execution Premium: Linking Strategy to Operations for Competitive Advantage. Harvard Business Press, pp. 336, (2008)

Larrosa, A. Strategor, Estrategia, Estructura, Decisión, Identidad. Tercera Edición, pp. 3-596, Editorial Masson S.A., Barcelona, España (1995)

Modelo Educativo de la Universidad del Bío-Bío, Documento Institucional (2008)

Ohmae, K., The Mind of Strategist: The Art of Japanese Business, pp. 1- 282, Editorial McGraw Hill, New York, Estados Unidos (2004)

Orientaciones para implementación del Modelo Educativo en el marco de la Renovación Curricular en la Universidad del Bío-Bío, Documento Institucional (2010)

Pettit, P. El diseño institucional y la elección racional. En Teoría del diseño institucional. Compendiado por Robert Goodin. Editorial Gedisa, Barcelona, España (2003)

Plan General de Desarrollo Universitario 2011-2014, Universidad del Bio Bio, Chile. Documento Institucional, http://www.ubiobio.cl (2011)

Quinn, J. Strategic goals: process and politics, Sloan Management Review, pp. 21-37, (1977)

RAE. [en línea] Real Academia Española. www.rae.es. www.casadellibro.com. Revisado el 12 mayo 2014

Richardson, B. y Richardson, R. Planeación de negocios: un enfoque de administración estratégica. Primera Edición, Editorial Continental, México DF, México (1996)

Rodríguez Valencia. Introducción a la administración con enfoque de sistemas, Tercera Edición, pp. 1-730, Editorial ECAFSA, México DF, México. (1998)

Senge, P. La Quinta Disciplina. Cómo impulsar el aprendizaje en la organización inteligente. S.A. Ediciones Granica, Barcelona, España (1999)

Taylor, J.W. Planificación Estratégica para la empresa de éxito, pp. 210, Editorial Modern Business Report, Nueva York, Estados Unidos (1979)

Teece, D., Pisano, G. y Shuen, A. Dynamic Capabilities and Strategic Management, Strategic Management Journal, Vol.18, No. 7, pp. 509-533 (1997)

Thompson, A. y Strickland, A. Strategic Management: Concept and Cases. Undécima Edición, Editorial McGraw Hill, Irwin, Estados Unidos (1999) 